\title{
OPEN Free-water diffusion tensor imaging improves the accuracy and sensitivity of white matter analysis in Alzheimer's disease
}

\begin{abstract}
Maurizio Bergamino ${ }^{1}$, Ryan R. Walsh ${ }^{2}$ \& Ashley M. Stokes ${ }^{1 凶}$
Magnetic resonance imaging (MRI) based diffusion tensor imaging (DTI) can assess white matter (WM) integrity through several metrics, such as fractional anisotropy (FA), axial/radial diffusivities (AxD/RD), and mode of anisotropy (MA). Standard DTI is susceptible to the effects of extracellular free water (FW), which can be removed using an advanced free-water DTI (FW-DTI) model. The purpose of this study was to compare standard and FW-DTI metrics in the context of Alzheimer's disease (AD). Data were obtained from the Open Access Series of Imaging Studies (OASIS-3) database and included both healthy controls $(\mathrm{HC})$ and mild-to-moderate $A D$. With both standard and FW-DTI, decreased FA was found in AD, mainly in the corpus callosum and fornix, consistent with neurodegenerative mechanisms. Widespread higher AxD and RD were observed with standard DTI; however, the FW index, indicative of AD-associated neurodegeneration, was significantly elevated in these regions in $A D$, highlighting the potential impact of free water contributions on standard DTI in neurodegenerative pathologies. Using FW-DTI, improved consistency was observed in FA, $A x D$, and $R D$, and the complementary FW index was higher in the AD group as expected. With both standard and FW-DTI, higher values of MA coupled with higher values of FA in AD were found in the anterior thalamic radiation and cortico-spinal tract, most likely arising from a loss of crossing fibers. In conclusion, FW-DTI better reflects the underlying pathology of AD and improves the accuracy of DTI metrics related to WM integrity in Alzheimer's disease.
\end{abstract}

Alzheimer's disease (AD) is a neurodegenerative disorder and the most common cause of dementia in older adults ${ }^{1}$. Current biomarkers for AD target the dominant pathological paradigm, characterized by beta-amyloid and tau pathologies, as well as neurodegenerative changes. While much of the pathologic evidence of AD is found in gray matter, it is well recognized that concomitant white matter (WM) alterations occur in the brains of $A D$ populations, suggesting an expanded role for WM-based biomarkers in $\mathrm{AD}^{2}$.

Diffusion-based magnetic resonance imaging (dMRI) enables the in vivo evaluation of brain microstructure and, more specifically, WM integrity by probing water diffusivity associated with axons ${ }^{3}$; most commonly, diffusion-related parameters are obtained by analysis using the diffusion tensor imaging (DTI) model. In WM, faster diffusivity along the fibers, with lower perpendicular diffusivity, yields a higher fractional anisotropy (FA). While decreased FA has been associated with demyelination, edema, gliosis, and inflammation, FA is unfortunately an indirect marker of WM integrity because the individual microscopic contributions of various non-WM components cannot be disentangled ${ }^{4,5}$. Other DTI-derived metrics include axial diffusivity $(\mathrm{AxD})$, which may be related to axonal damage, and radial diffusivity (RD), which is correlated with myelin integrity, axonal diameter and density, and fiber coherence ${ }^{6}$. Prior DTI studies have shown that AD is associated with lower FA and higher $\mathrm{AxD}$ and $\mathrm{RD}$ values across multiple regions in the $\mathrm{WM}$, including the corpus callosum (CC), fornix, cingulum, inferior longitudinal fasciculus (ILF), and superior longitudinal fasciculus (SLF) ${ }^{7-9}$. Moreover, changes in DTI metrics may precede both neuronal loss and symptoms onset, suggesting their potential role as early biomarkers. While several studies have shown a correlation between DTI-derived metrics and cognitive scores ${ }^{10-12}$, other similar investigations have failed to show a significant correlation ${ }^{13-16}$ suggesting methodologic and/or analytic impacts on dMRI-derived data.

\footnotetext{
${ }^{1}$ Division of Neuroimaging Research, Barrow Neurological Institute, Phoenix, AZ 85013, USA. ${ }^{2}$ Muhammad Ali Parkinson Center, Barrow Neurological Institute, Phoenix, AZ 85013, USA. email: ashley.stokes@ barrowneuro.org
} 


\begin{tabular}{|l|l|l|l|l|l|}
\hline & N subjects & N females & Age & MMSE & CDR \\
\hline HC & 30 & 17 & $73(6)$ & $29.10(1.24)$ & $0(0)$ \\
\hline AD & 28 & 15 & $75(7)$ & $24.18(5.02)$ & $0.82(0.55)$ \\
\hline$t$-test & & & $\mathrm{t}=-1.51 ; p=0.137$ & $\mathrm{t}=-5.21 ; p<0.0001^{*}$ & $\mathrm{t}=-8.22 ; p<0.0001^{*}$ \\
\hline CDR & HC & AD & \\
\hline CDR 0 (none) & 30 & 0 & \\
\hline CDR 0.5 (very mild) & 0 & 16 & \\
\hline CDR 1 (mild) & 0 & 10 & \\
\hline CDR 2 (moderate) & 0 & 1 & 1 \\
\hline CDR 3 (severe) & 0 & & 1 & \\
\hline
\end{tabular}

Table 1. Group demographics with MMSE and CDR scores. ${ }^{\star}$ Student t-test demonstrated differences in MMSE and CDR between AD and HC cohorts.

Although dMRI is a well-recognized method for assessing WM, it has several limitations, including known inaccuracies related to partial volume effects (PVEs), wherein the resulting DTI metrics reflect a weighted average of multiple diffusion components within a voxel and are thus no longer specific to a single tissue type ${ }^{17}$. The impact of PVEs may be particularly profound in $\mathrm{AD}$, where neuronal loss leads to secondary ex vacuo increases in cerebrospinal fluid (CSF) and free water (FW) across the brain. To overcome the effects of extracellular FW on DTI-derived metrics, a FW correction algorithm has been developed to quantify and remove the contribution of extracellular FW ${ }^{18}$. FW-DTI has been shown to improve both tissue specificity ${ }^{19}$ and DTI-based tract reconstruction ${ }^{18}$. This model has previously been used to study several neurological diseases, including $\mathrm{AD}^{20-24}$, Parkinson's disease ${ }^{25}$, and depression ${ }^{26,27}$.

Using FW-DTI in a healthy aging population enriched for AD risk, Hoy et al. found that DTI-based markers of altered microstructure were significantly associated with CSF biomarkers of preclinical AD pathology ${ }^{20}$. FW correction may be particularly important in the fornix, due to both its proximity to the lateral ventricles and its association with the hippocampus ${ }^{22}$, and may also improve sensitivity to conversion to mild cognitive impairment (MCI) relative to uncorrected DTI metrics ${ }^{23}$. The FW index has separately been proposed as a standalone biomarker of $\mathrm{AD}$-related pathology. This index has been associated with cognitive decline in multiple domains and may enable separation of vascular and neurodegenerative contributions to cognitive decline in the presence of mixed dementia ${ }^{21}$. More recently, an elevated FW index was observed in MCI and AD patients compared with healthy controls (HC), even after correction for white matter hyperintensities ${ }^{28}$. However, none of these studies have directly compared standard and FW-DTI metrics in the context of distinguishing between AD and HC. As $\mathrm{AD}$-related WM degeneration leads to a significant intravoxel FW component, FW-DTI may improve sensitivity to early brain changes and provide a clearer and more specific interpretation of results compared to standard DTI.

In this study, we compared FA, AxD, RD, and MA from both standard DTI and FW-DTI between AD and HC subjects obtained from the Open Access Series of Imaging Studies (OASIS-3) brain project ${ }^{29}$. This dataset was previously used to investigate $\mathrm{AD}$-related changes in the size and shape of the $\mathrm{CC}^{30,31}$. We also investigated the complementary FW index for both groups and analyzed voxel-based correlations between all DTI-derived metrics (from DTI and FW-DTI) and the mini-mental state examination (MMSE) score ${ }^{32}$. We hypothesize that FW-DTI will improve the sensitivity and specificity for detection of WM tract abnormalities in AD. More specifically, increased FW due to neurodegeneration reduces the accuracy of diffusion-derived metrics, and thus, the removal of FW contributions improves the estimation of DTI metrics and may increase confidence in the patterns observed across DTI studies. In addition, the ability to quantify FW may yield complementary insight into sub-voxel WM neurodegenerative processes. The application of FW-DTI for AD better reflects the underlying pathology and should thus further improve interpretation of AD WM pathology.

\section{Materials and methods}

Subjects. All data were downloaded from the OASIS-3 brain project (http://oasis-brains.org/), which is a neuroimaging dataset for normal aging and Alzheimer's disease and is freely available to the scientific community. Inclusion criteria were participants between the ages of 60 and 90 years who had 3 T dMRI data available with more than 30 directions. Exclusion criteria were cognitively impaired participants with dementia not attributed to $\mathrm{AD}$, healthy control participants with significant comorbidities, and participants for whom the MMSE was not available. Only one time-point was used per subject. Subsequently, this study included $30 \mathrm{HCs}$ (17 females; age (standard deviation, S.D.) $=73$ (6) years) and 28 AD subjects (primarily mild AD; 15 females; age $=75$ (7) years). Four AD subjects had remote mood disorders and one had a remote hypothyroidism as secondary medical diagnoses. The Clinical Dementia Rating $(\mathrm{CDR})^{33}$, which is a 5 -point scale used to characterize multiple domains of cognitive and functional abilities, was zero for all HCs and between 0.5 and 3.0 for the AD group. The MMSE is a 30-point assessment commonly used to measure cognitive impairment. The mean value of MMSE was 29.10 (S.D. 1.24) for HC and 24.18 (S.D. 5.02) for AD. All subject characteristics are summarized in Table 1.

MRI protocol for DTI. All dMRI data collected from the OASIS-3 database were acquired using a 3 T scanner (Siemens). For all subjects, the acquisition was performed using 64 diffusion-encoding directions (b value: 
$1000 \mathrm{~s} / \mathrm{mm}^{2}$; TR/TE: $11,000 / 87.0 \mathrm{~ms}$; flip-angle =90'; matrix: $96 \times 96$; field of view (FOV): $24.0 \times 24.0 \mathrm{~cm}$; slice thickness: $2.5 \mathrm{~mm}$; 64 axial slices) and one non-diffusion-weighted image (B0 image).

Data preprocessing. DMRI data were downloaded in NIFTI format and processed as previously described $^{26,27}$. Briefly, the data were preprocessed using the functional magnetic resonance imaging of the brain (FMRIB) software library tool (FSL, version 5.0.4) $)^{34}$. Following translation and rotation estimation, the raw dMRI images were corrected for eddy currents and motion ${ }^{35}$. After motion correction, the gradient orientations were compensated (prior to calculating B-matrices) to account for the rotational component of registration. A brain mask was defined for each subject using the Brain Extraction Toolbox (BET) ${ }^{36}$ for the single B0 images. DTI standard (uncorrected for FW) and FW-corrected maps were calculated using an in-house MATLAB script ${ }^{26}$, which is available upon request. The FW maps were computed by fitting the following model at each voxel ${ }^{18}$ :

$$
A_{g}(D, f)=f \cdot\left(\exp \left[-b g^{T} D g\right]\right)+(1-f) \cdot \exp \left[-b D_{\text {water }}\right]
$$

where $A_{g}$ is the modeled attenuated signal (normalized by B0) for the applied diffusion gradient $g$, and $b$ is the b-value $\left(1000 \mathrm{~s} / \mathrm{mm}^{2}\right)$. The first term reflects the tissue compartment, where $D$ is the diffusion tensor of this compartment, $f$ is the fractional volume of the compartment, and $g^{T}$ is the transpose of the vector $g$. The second term reflects an isotropic free-water compartment with a fractional volume of $(1-f)$, where the diffusion coefficient $D_{\text {water }}$ is set to the diffusivity of water at body temperature $\left(3 \times 10^{-3} \mathrm{~mm}^{2} / \mathrm{s}\right)$. AxD, RD, and FA were obtained from $D$. Standard DTI metrics are reported as $\mathrm{AxD}, \mathrm{RD}$, and $\mathrm{FA}$, while FW-corrected DTI metrics are reported as $A x D_{t}, R D_{t}$, and $F A_{t}$, where the $t$ is indicative of greater tissue specificity from FW-DTI.

While FA indicates deviation from a spherical-shaped tensor ( $F A=0$; isotropic diffusion), the mode of anisotropy (MA) is a complementary measure that discriminates between linear and planar anisotropy. MA is mathematically orthogonal to FA and relates to the skewness of the DTI eigenvalues $\left(\lambda_{1}, \lambda_{2}, \lambda_{3}\right)^{37}$ :

$$
M A=\frac{\left(-\lambda_{1}-\lambda_{2}+2 \lambda_{3}\right)\left(2 \lambda_{1}-\lambda_{2}-\lambda_{3}\right)\left(-\lambda_{1}+2 \lambda_{2}-\lambda_{3}\right)}{2\left(\lambda_{1}^{2}+\lambda_{2}^{2}+\lambda_{3}^{2}-\lambda_{1} \lambda_{2}-\lambda_{1} \lambda_{3}-\lambda_{2} \lambda_{3}\right)^{3 / 2}}
$$

After quantifying all DTI-derived metrics, the B0 images from each subject were used to create a B0 groupwise template image using buildtemplateparallel.sh included in ANTs; this procedure has been demonstrated to improve the final normalization procedure ${ }^{38}$. The B0 group-wise template image was subsequently normalized to the IIT_mean_b0 image by ANTs symmetric image normalization (SyN) algorithm ${ }^{39}$. The warp fields obtained from this normalization and from the B0 group-wise template creation were used to transfer all DTI maps (standard and FW-corrected) to the Illinois Institute of Technology (IIT) Human Brain Atlas (v.4.1), which contains both anatomical and DTI brain templates in International Consortium for Brain Mapping (ICBM)-152 space ${ }^{40}$. All DTI maps in standard space were smoothed using FSL with an isotropic Gaussian kernel (sigma, $3 \mathrm{~mm}$ ).

Statistical analysis. Age, MMSE, and CDR scores are presented as mean and standard deviation (SD) for each group. Differences in age, sex, and cognitive test scores between groups were assessed using the Student's t-test. Comparisons of all DTI-, FW-DTI- metrics, and FW maps between groups were performed using the FSL Randomise tool ${ }^{41}$ with threshold-free cluster enhancement (TFCE) ${ }^{42}$ and a correction for multiple comparisons via Family-Wise Error (FWE) rate ${ }^{38}$ at a 0.01 level. A WM mask was defined using a threshold of 0.2 on the average values of FA (from standard DTI) and was applied to the other DTI maps. For each voxel, the DTI-derived metrics were fitted by an Analysis of Covariance model (ANCOVA), which included age and sex as covariates. The number of randomized permutations was set at 5,000. The clusters identified to be statistically different between groups were labeled according to the JHU DTI-based white-matter atlases ${ }^{43,44}$. All results are reported with a significance threshold of $p$ value $<0.01$, corrected for FWE, except where otherwise noted.

For the cognitively impaired group (AD only), the voxel-based relationship between MMSE scores and DTI metrics was assessed through Randomise with significance at $p$-value $<0.01$ (FWE-corrected).

\section{Results}

Student's t-test did not show any difference in age $(\mathrm{t}=-1.51 ; p=0.137)$ or $\operatorname{sex}(\mathrm{t}=0.233 ; p=0.817)$ between HC and AD. Significant differences were found for MMSE $(t=-5.21 ; p<0.0001)$ and for CDR $(t=-8.22 ; p<0.0001)$, as shown in Table 1.

Standard DTI. Figure 1 shows the clusters of significant differences between the two groups obtained with standard DTI metrics with the corresponding violin plots quantifying the DTI differences for each group (across all combined clusters). For FA (panel (a)), lower FA was observed in AD compared with HC mainly in the fornix (cluster covering 93.3\% of the fornix) and CC (covering 35.5\% of CC). Small clusters where AD had higher FA values than $\mathrm{HC}$ were found in the right anterior thalamic radiation (ATR), cortical spinal tract (CST), posterior limb of internal capsule (PLIC), and superior corona radiata (SCR). More widespread differences between HC and $\mathrm{AD}$ were observed using $\mathrm{AxD}$ and $\mathrm{RD}$ (panel (b) and (c), respectively). Additionally, all significant clusters corresponded to higher values of $\mathrm{AxD}$ and $\mathrm{RD}$ metrics in the $\mathrm{AD}$ group, with no clusters corresponding to lower $\mathrm{AxD}$ or RD.

For each brain region, the significant cluster sizes (as percent volume) are reported in Table 2. Several clusters were observed where multiple DTI metrics were simultaneously altered between HC and AD groups, as shown in Supplementary Table 1. Regions where lower FA values overlapped with higher AxD and RD included the fornix, CC, forceps minor, and left cingulum. Higher FA values were found to overlap with higher AxD in 


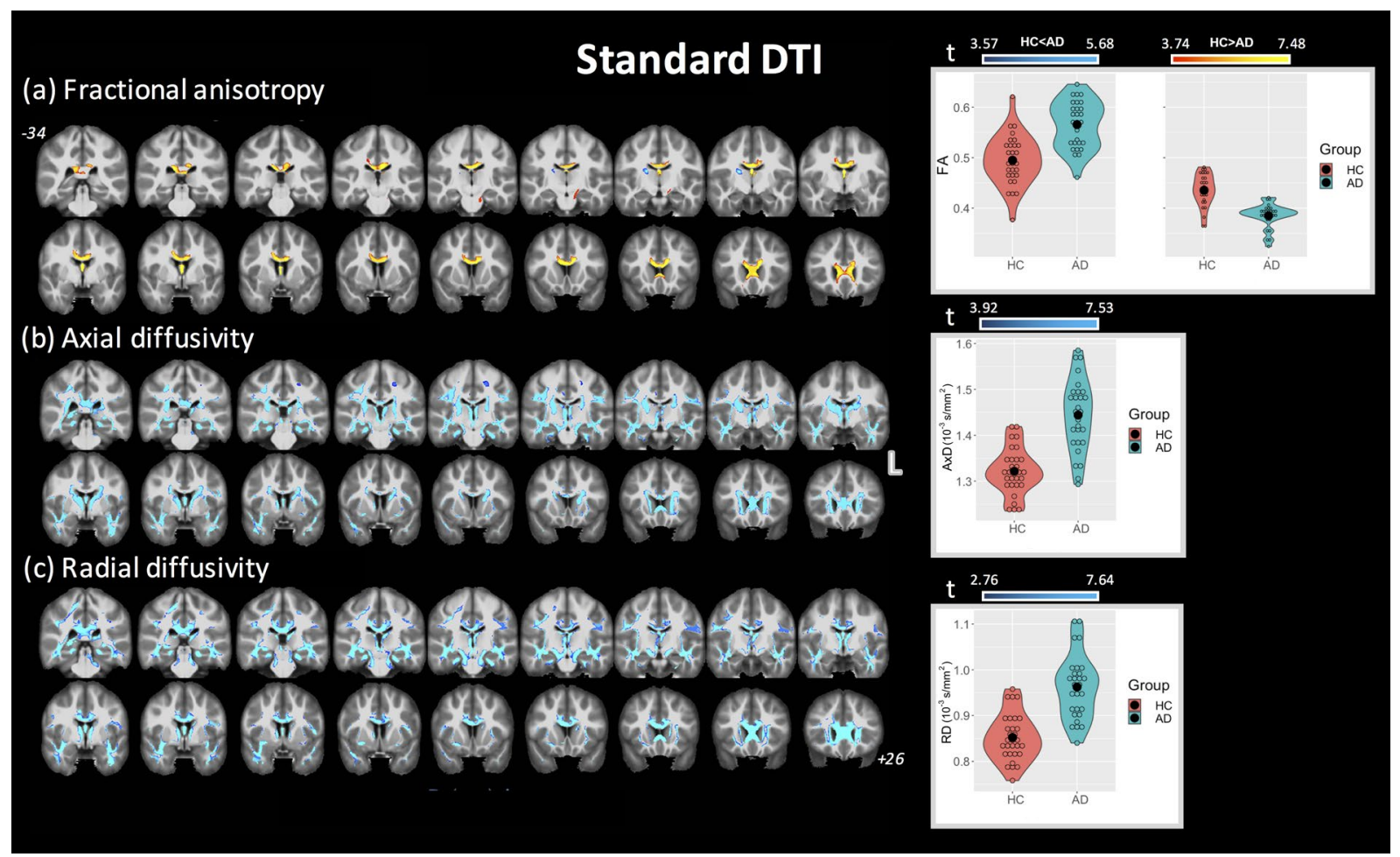

Figure 1. Coronal view of ANCOVA analysis between $\mathrm{HC}$ and $\mathrm{AD}$ subjects for standard DTI metrics. Clusters are reported at a significance threshold of $p$-value $<0.01$ corrected for FWE. (a) FA, (b) AxD, and (c) RD analysis. The color bars indicate values that are lower in AD than HC (red-to-yellow) and higher in AD than HC (blue-to-cyan). For axial and radial diffusivities, only clusters with higher values in AD than $\mathrm{HC}$ were found. The corresponding violin plots represent the DTI metrics inside the significant clusters for each group.

the right PLIC, CST, and SCR. Substantial overlap was observed for clusters with higher AxD and higher RD, including $85 \%$ of the cingulum (hippocampus), $76 \%$ of the fornix, and $49 \%$ of the sagittal stratum. Finally, Supplementary Table 2 shows the corresponding mean DTI values inside each significant cluster for regions with higher and lower values for each group.

FW-DTI. Figure 2 shows the clusters of significant differences between groups for FW-DTI metrics with the corresponding violin plots quantifying the DTI differences for each group (across all combined clusters), where $\mathrm{FA}_{\mathrm{t}}, \mathrm{AxD}_{\mathrm{t}}$, and $\mathrm{RD}_{\mathrm{t}}$ are displayed in panels (a), (b), and (c) (at $p<0.01 \mathrm{FWE}$ corrected, unless otherwise indicated). The clusters where $\mathrm{AD}$ had lower $\mathrm{FA}_{\mathrm{t}}$ values than $\mathrm{HC}$ correspond to the same WM locations as those observed using standard FA (specifically, fornix and CC); however, these clusters (covering $71 \%$ and $19.2 \%$ of fornix and CC, respectively) are smaller than standard FA (93.3\% and 35.5\%, respectively). Higher $\mathrm{FA}_{\mathrm{t}}$ values in AD compared with HC were found bilaterally for ATR, CST, limb of internal capsule (LIC), SCR, and superior fronto-occipital fasciculus (SFOF). With regard to $\mathrm{AxD}_{\mathrm{t}}$ and $\mathrm{RD}_{\mathrm{t}}$, both higher and lower values were observed in multiple regions in the $\mathrm{AD}$ group (recall that only higher $\mathrm{AxD}$ and $\mathrm{RD}$ were observed using standard $\mathrm{DTI}$ ). More specifically, $\mathrm{AxD}_{\mathrm{t}}$ was lower in the $\mathrm{AD}$ group in CC and fornix and predominantly higher in the ATR, CST, and retrolenticular part of internal capsule. Lower $\mathrm{RD}_{\mathrm{t}}$ was observed with $\mathrm{AD}$ mainly in the ATR, CST, PLIC, and SFOF, corresponding to similar areas with higher $\mathrm{FA}_{\mathrm{t}}$. A small cluster inside the body of the CC showed higher $\mathrm{RD}_{\mathrm{t}}$ in the $\mathrm{AD}$ group, albeit at $p<0.05 \mathrm{FWE}$ corrected.

For each brain region, the significant cluster sizes (as percent volume) are reported in Table 2. As for standard DTI, several clusters were observed where multiple DTI metrics were simultaneously altered between HC and AD groups, as shown in Supplementary Table 1. No regions were observed where lower $F A_{t}$ values overlapped with higher $\mathrm{AxD}_{t}$ and $\mathrm{RD}_{\mathrm{t}}$; however, lower $\mathrm{FA}_{t}$ values overlapped with lower $\mathrm{AxD}_{t}$ and $\mathrm{RD}_{t}$ values in the fornix (4.8\%), while lower $\mathrm{FA}_{t}$ corresponded with lower $\mathrm{AxD}_{t}$ in several regions including the $\mathrm{CC}$ and forceps minor. Higher $\mathrm{FA}_{t}$ values were found to overlap with higher $\mathrm{AxD}_{\mathrm{t}}$ and lower $\mathrm{RD}_{\mathrm{t}}$ in the SFOF, right PLIC, SCR, and anterior LIC. Several clusters were observed with higher $\mathrm{AxD}_{t}$ and lower $\mathrm{RD}_{\mathrm{t}}$, including the left CST, right PLIC, and right SCR. Unlike standard DTI, no clusters demonstrated simultaneously higher $\mathrm{AxD}_{t}$ and higher $\mathrm{RD}_{\mathrm{t}}$. Finally, Supplementary Table 3 shows the corresponding mean FW-DTI values inside each significant cluster for regions with higher and lower values for each group.

FW-index. Figure 3 shows the significant differences between groups using the FW index (obtained from the fit in Eq. 1) at $p<0.01 \mathrm{FWE}$ corrected. The corresponding violin plots are also shown for FW values for each group (across all combined clusters). Only clusters with higher FW values in AD compared with HC were found, 


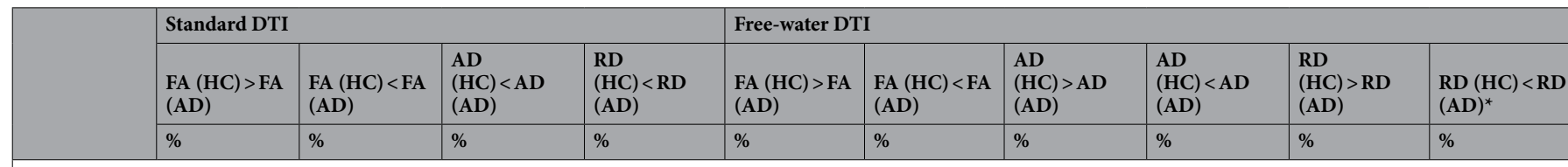

\section{JHU WM tract. atlas}

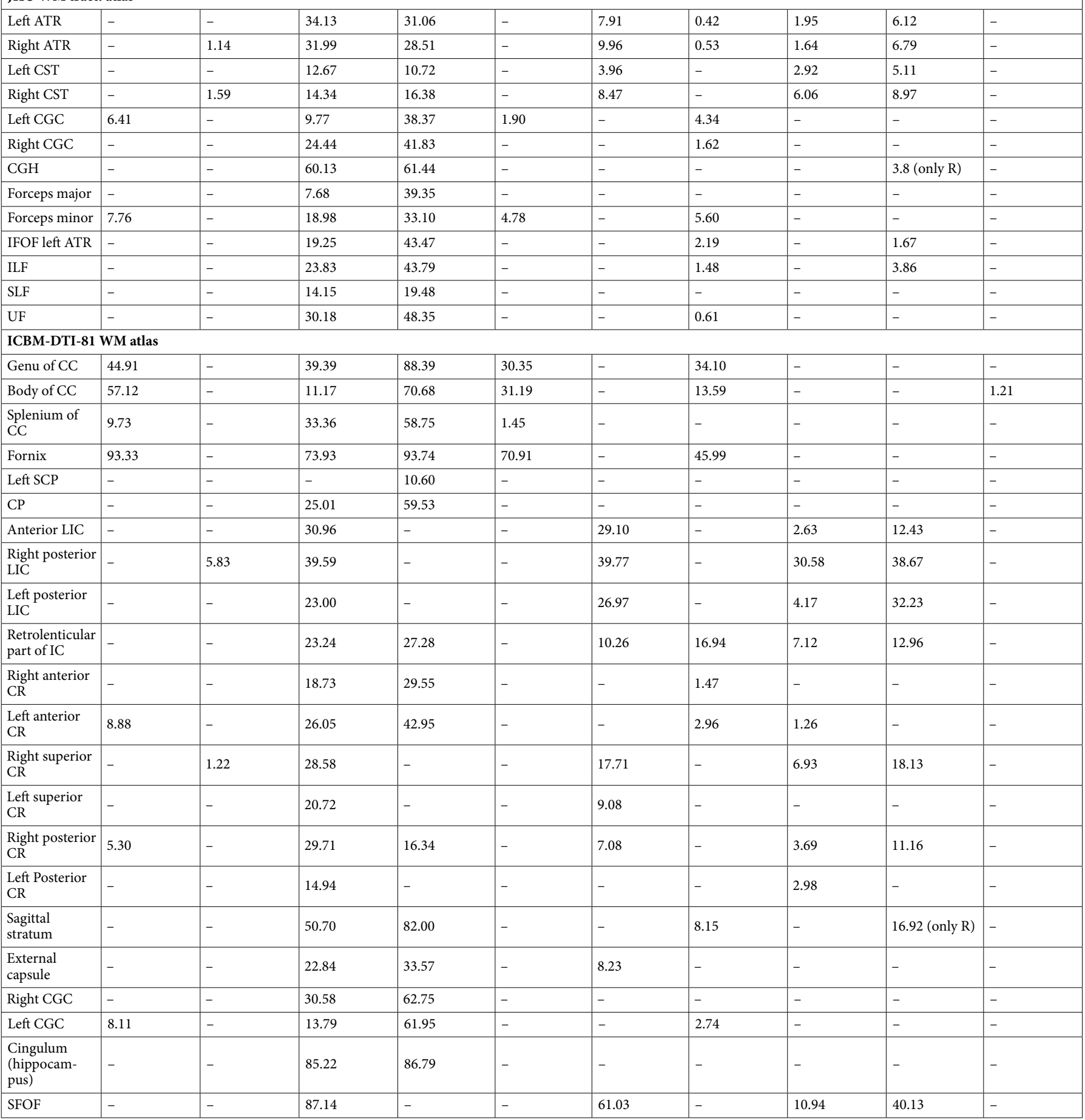

Table 2. Brain locations where clusters of significant differences between groups were found for both standard and FW-DTI analyses. All results are shown for $p<0.01$ (FWE corrected), except for the last column, indicated by ${ }^{*}$, where $\mathrm{RD}(\mathrm{HC})<\mathrm{RD}(\mathrm{AD})$ is reported at $p<0.05$ (FWE corrected). \% columns indicate the percent volume of the cluster inside the corresponding brain area. For JHU white-matter tractography atlas: ATR anterior thalamic radiation, CST cortical spinal tract, $C G C$ cingulum (cingulate gyrus part), CGH cingulum (hippocampal part), IFOF inferior fronto-occipital fasciculus, ILF inferior longitudinal fasciculus, $U F$ uncinate fasciculus. For ICBM-DTI-81 white-matter atlas: CC corpus callosum, LIC limb of internal capsule, IC internal capsule, $C R$ corona radiate, $S S$ sagittal stratum, SFOF superior fronto-occipital fasciculus. 


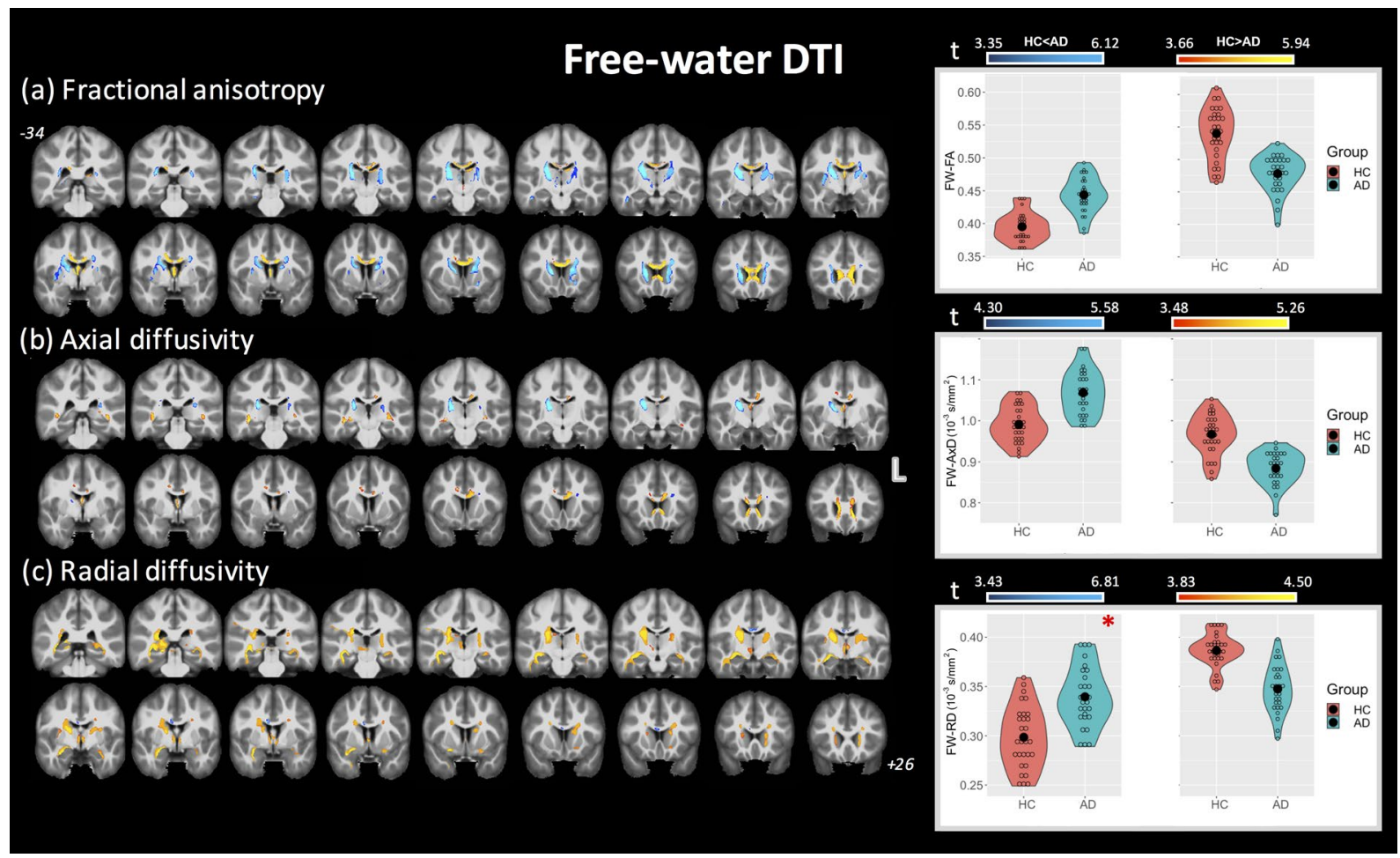

Figure 2. Coronal view of ANCOVA analysis between $\mathrm{HC}$ and $\mathrm{AD}$ subjects for the FW-DTI metrics. Clusters are reported at a significance threshold of $p$-value $<0.01$ corrected for $F W E$, except for $R_{t}(H C)<R D_{t}(A D)$, which is reported at $p$-value $<0.05 \mathrm{FWE}$ - corrected (c) and is indicated by the *. (a) $\mathrm{FA}_{\mathrm{t}}$, (b) $\mathrm{AxD}_{\mathrm{t}}$, and (c) $\mathrm{RD}_{\mathrm{t}}$ analysis. The color bars indicate values that are lower in AD than HC (red-to-yellow) and higher in AD than HC (blue-to-cyan). The corresponding violin plots represent the FW-DTI metrics inside the significant clusters for each group.

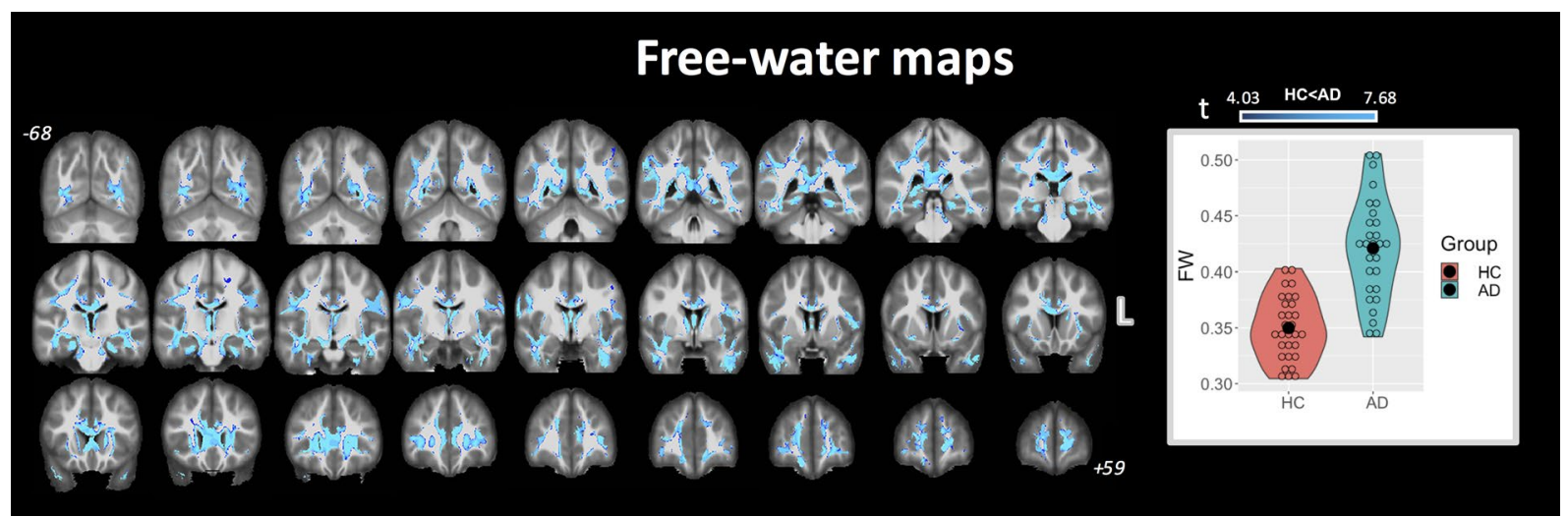

Figure 3. Coronal view of ANCOVA analysis between $\mathrm{HC}$ and $\mathrm{AD}$ subjects for $\mathrm{FW}$ index. Clusters are reported at a significance threshold of $p$-value $<0.01$ corrected for FWE. Higher FW in AD compared to HC was observed widespread across cerebral WM. No significant clusters were found with FW (HC) $>$ FW (AD). The corresponding violin plots represent the FW metrics inside the significant clusters for each group.

with clusters covering $34 \%$ of the total WM mask. These differences were observed in several WM regions, including most prominently the fornix, cingulum (both hippocampal and cingulate gyrus parts), and CC. The complete list of group-wise significant clusters for the FW index between HC and AD is reported in Table 3.

MA-index. The results for MA are reported in Fig. 4. Significantly higher values of MA (from both standard DTI and FW-DTI) in AD compared with HC were observed in the right CST, right PLIC, right SCR, and right SFOF, corresponding to more linear anisotropy. On the other hand, lower MA values (from standard DTI and FW-DTI) in AD compared with HC were found mainly in the genu of CC and in the external capsule (EC), corresponding to more planar anisotropy. It should be noted that similar results for MA were obtained with both standard DTI and FW-DTI analysis. The complete list of group-wise significant clusters is reported in Table 4. 


\begin{tabular}{|c|c|c|c|}
\hline & $\%$ & FW (HC) & FW (AD) \\
\hline \multicolumn{4}{|l|}{ JHU WM tract. atlas } \\
\hline ATR & 31.39 & $0.36(0.03)$ & $0.42(0.04)$ \\
\hline CST & 12.98 & $0.30(0.02)$ & $0.35(0.02)$ \\
\hline CGC & 56.34 & $0.28(0.03)$ & $0.33(0.04)$ \\
\hline CGH & 85.16 & $0.33(0.03)$ & $0.41(0.06)$ \\
\hline Forceps major & 40.10 & $0.33(0.03)$ & $0.38(0.05)$ \\
\hline Forceps minor & 39.19 & $0.34(0.04)$ & $0.40(0.04)$ \\
\hline IFOF & 39.92 & $0.28(0.03)$ & $0.32(0.04)$ \\
\hline ILF & 52.05 & $0.27(0.03)$ & $0.32(0.05)$ \\
\hline SLF & 30.08 & $0.28(0.03)$ & $0.33(0.04)$ \\
\hline UF & 47.81 & $0.28(0.03)$ & $0.34(0.04)$ \\
\hline \multicolumn{4}{|l|}{ ICBM-DTI-81 WM atlas } \\
\hline Genu of CC & 67.27 & $0.39(0.03)$ & $0.45(0.04)$ \\
\hline Body of CC & 47.89 & $0.37(0.04)$ & $0.43(0.03)$ \\
\hline Splenium of CC & 52.17 & $0.30(0.04)$ & $0.36(0.04)$ \\
\hline Fornix & 95.62 & $0.72(0.06)$ & $0.81(0.05)$ \\
\hline $\mathrm{CP}$ & 41.40 & $0.33(0.03)$ & $0.38(0.03)$ \\
\hline Left anterior LIC & 10.09 & $0.25(0.03)$ & $0.30(0.04)$ \\
\hline Right retrolenticular part of IC & 21.22 & $0.28(0.03)$ & $0.32(0.03)$ \\
\hline Anterior CR & 29.23 & $0.25(0.04)$ & $0.30(0.04)$ \\
\hline Right posterior CR & 19.87 & $0.26(0.03)$ & $0.31(0.04)$ \\
\hline SS & 65.31 & $0.29(0.02)$ & $0.34(0.04)$ \\
\hline EC & 30.24 & $0.26(0.03)$ & $0.31(0.04)$ \\
\hline $\mathrm{CGH}$ & 90.64 & $0.34(0.03)$ & $0.41(0.06)$ \\
\hline
\end{tabular}

Table 3. Brain locations where clusters of significant differences between groups were found for FW maps analysis at $p<0.01$ (FWE corrected). \% columns indicate the percent volume of the cluster inside the corresponding brain area. Only clusters with higher FW values in AD than HC were observed. For JHU whitematter tractography atlas: ATR anterior thalamic radiation, CST cortical spinal tract, CGC cingulum (cingulate gyrus part), $C G H$ cingulum (hippocampal part), IFOF inferior fronto-occipital fasciculus, ILF inferior longitudinal fasciculus, $S L F$ superior longitudinal fasciculus, $U F$ uncinate fasciculus. For ICBM-DTI-81 whitematter atlas: $C C$ corpus callosum, $C P$ cerebral peduncle, $L I C$ limb of internal capsule, $I C$ internal capsule, $C R$ corona radiata, $S S$ sagittal stratum, EC external capsule.

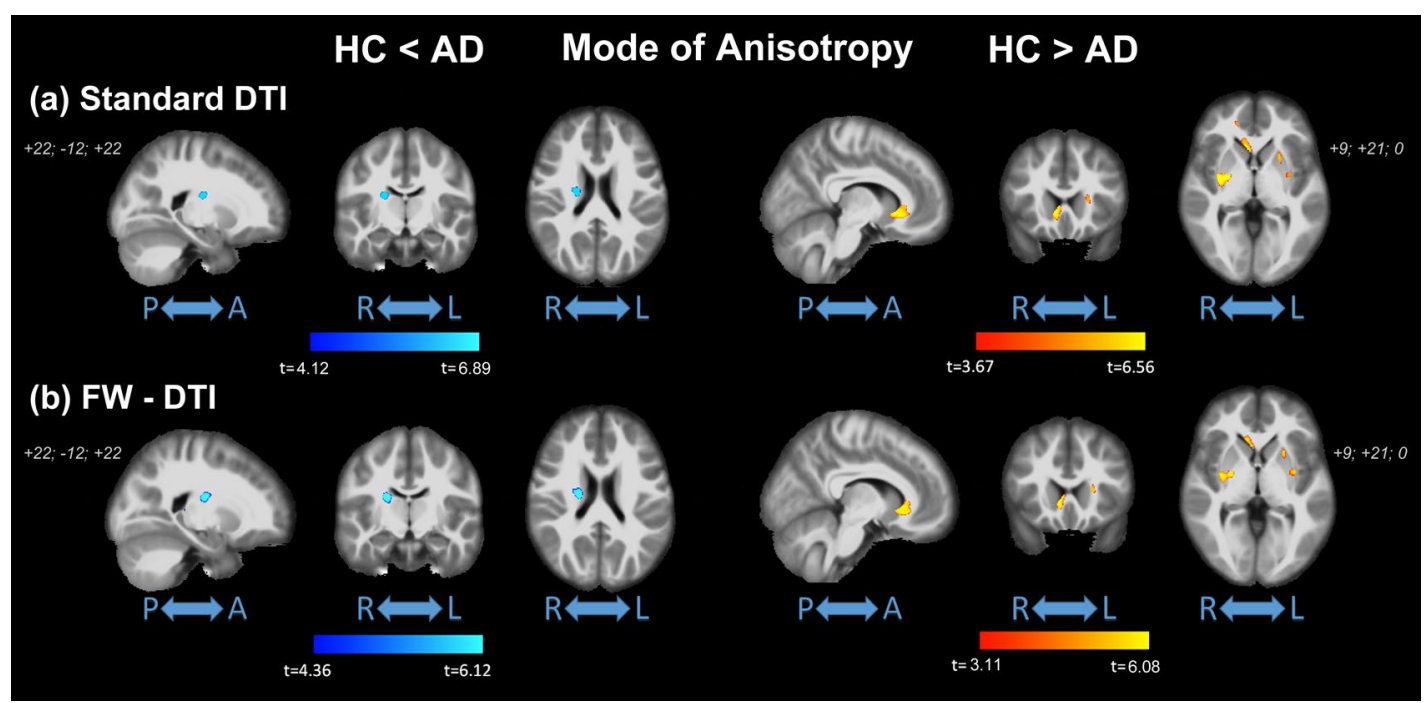

Figure 4. Coronal view of ANCOVA analysis between $\mathrm{HC}$ and $\mathrm{AD}$ subjects for MA index from (a) standard DTI and (b) FW-DTI. Left: MA in $\mathrm{HC}<\mathrm{MA}$ in $\mathrm{AD}$; right: MA in $\mathrm{HC}>\mathrm{MA}$ in $\mathrm{AD}$. Clusters are reported at a significance threshold of $p$-value $<0.01$ corrected for FWE. 


\begin{tabular}{|l|l|l|l|l|}
\hline \multirow{2}{*}{} & \multicolumn{5}{l}{ Standard MA } & FW-MA & \multicolumn{2}{l|}{} \\
\cline { 2 - 6 } & HC $<$ AD & HC $>$ AD & HC $<$ AD & HC $>$ AD \\
\cline { 2 - 6 } & $\%$ & $\%$ & $\%$ & $\%$ \\
\hline JHU WM tract. atlas & - & 0.87 & - & 0.50 \\
\hline Left ATR & 0.52 & - & 0.76 & - \\
\hline Right CST & - & 0.42 & - & - \\
\hline Forceps major & - & 1.73 & - & 0.87 \\
\hline Forceps minor & - & 1.12 & - & 0.60 \\
\hline IFOF & - & 0.51 & - & - \\
\hline Left UF & - & 3.31 & - & 1.97 \\
\hline Right UF & - & & & \\
\hline ICBM-DTI-81 WM atlas & - & 10.07 & - & 6.76 \\
\hline Genu of CC & - & 4.24 & - & 2.61 \\
\hline Left anterior LIC & 1.61 & - & 2.81 & - \\
\hline Right posterior LIC & - & 1.29 & - & - \\
\hline Right anterior CR & - & 1.70 & - & 0.68 \\
\hline Left anterior CR & 2.34 & - & 2.87 & - \\
\hline Right superior CR & - & 10.40 & - & 6.26 \\
\hline EC & & & & \\
\hline
\end{tabular}

Table 4. Brain locations where clusters of significant differences between groups were found for MA index analysis at $p<0.01$ (FWE corrected). \% columns indicate the percent volume of the cluster inside the corresponding brain area. For JHU white-matter tractography atlas: ATR anterior thalamic radiation, CST cortical spinal tract, IFOF inferior fronto-occipital fasciculus, $I L F$ inferior longitudinal fasciculus, $U F$ uncinate fasciculus. For ICBM-DTI-81 white-matter atlas: CC corpus callosum, LIC limb of internal capsule, CR corona radiata, EC external capsule, SFOF superior fronto-occipital fasciculus.

Voxel-based correlations between DTI/FW-DTI metrics and MMSE. No significant voxel-based correlation was found between MMSE and either standard or FW-DTI metrics at $p<0.05$ FWE corrected level.

Correlations between standard and FW-DTI metrics. Supplementary Fig. 1 shows the population scatter-plots for standard and FW-DTI metrics, while the group-wise correlations for HC and AD are provided in each plot. Panels (a) and (b) show the correlations between FA and AxD, while panels (c) and (d) show the correlations between FA and RD. It should be noted that the correlations increased after FW correction (e.g., for the $\mathrm{AD}$ group: the correlations between $\mathrm{FA}$ and $\mathrm{AxD}$ increased from 0.160 (standard) to 0.856 (FW-corrected) and from -0.464 (standard) to -0.803 (FW-corrected) between FA and RD). Similar increases were observed for both $\mathrm{HC}$ and $\mathrm{AD}$ groups, suggesting that older HC participants also have substantial FW contributions to their dMRI-derived metrics.

Panels (e)-(g) show the correlations between FA and FW-FA, $\mathrm{AxD}$ and $\mathrm{FW}-\mathrm{AxD}_{\mathrm{t}}, \mathrm{RD}$ and $\mathrm{FW}-\mathrm{RD}_{\mathrm{t}}$, respectively. By removing the isotropic diffusion contribution, the impact of FW-correction is to increase $\mathrm{FA}_{\mathrm{t}}$, decrease $\mathrm{AxD}_{\mathrm{t}}$, and decrease $\mathrm{RD}_{\mathrm{t}}$, relative to the corresponding standard DTI metrics. These panels also show that FA is less affected by the FW component than $\mathrm{AxD}$ and $\mathrm{RD}$ (i.e., the correlation for standard vs. FW FA is higher than that for $\mathrm{AxD}$ and $\mathrm{RD}$ ). As $\mathrm{AxD}$ and $\mathrm{RD}$ metrics are more influenced by free-water components (i.e., have lower correlations between standard and FW metrics), this is consistent with the widespread AxD and RD changes without FW-correction. Here again, there is not a strong group difference in the correlations. Additionally, the striking differences for $\mathrm{AxD}$ and $\mathrm{RD}$ metrics between standard and FW-correction can be attributed to their biophysical origin; that is, raw diffusivity parameters $\mathrm{AxD}$ and $\mathrm{RD}$ are likely to be more impacted by intravoxel free-water diffusion than the composite metric FA.

\section{Discussion}

In this study, WM integrity was assessed in patients with Alzheimer's disease using both standard and FWcorrected DTI metrics. Although DTI is a commonly used method for assessing WM, PVEs can reduce the accuracy of DTI metrics, ultimately limiting their interpretation. To mitigate PVE, free water-contaminated voxels were removed by incorporating a FW component in the conventional diffusion modeling pipeline ${ }^{18}$, producing more accurate FW-corrected DTI metrics and the complementary FW index. Using both standard and FW-DTI, significant WM differences were observed between $\mathrm{HC}$ and AD subjects in several regions, including both higher and lower FA. Both AxD and RD were highly impacted by the effects of FW, and FW-corrected AxDt and RDt were more consistent with pathology-based expected spatial patterns of neurodegeneration. The FW index was higher in $\mathrm{AD}$ than $\mathrm{HC}$ across many regions, as expected. The FW correction method has shown promise in many neurological diseases of the $\mathrm{WM}^{21,24-27}$, particularly those with neurodegenerative aspects; similarly, we now demonstrate that the application of FW correction in AD improves the accuracy and sensitivity of the resulting DTI metrics. 
Lower FA values, indicative of more isotropic motion, are consistent with neurodegenerative changes and were observed in $\mathrm{AD}$ in multiple regions, including most prominently the fornix and CC. Of particular interest, the fornix is the major output tract of the hippocampus and plays a major role in episodic memory ${ }^{2,45}$. In the context of $\mathrm{AD}$, pathological changes in the fornix may occur at an early disease stage before clinical manifestations. Lower FA and higher MD in the fornix has been a robust and consistent finding in $\mathrm{AD}^{46,47}$ and may correlate with cognitive decline ${ }^{46,48}$. Similarly, FA reductions in the fornix have been identified using skeletonized tractbased spatial statistics (TBSS) in early-symptomatic individuals and those at high risk for developing $\mathrm{AD}^{49-52}$. However, the fornix is particularly prone to PVEs due to its proximity to the lateral ventricles ${ }^{2}$, which may be further exacerbated by atrophy-related ventricular expansion in $\mathrm{AD}$. While decreases in standard FA may reflect a complex combination of atrophy and microstructural changes, these decreases remained after correction for FW, indicating that they reflect true pathological changes in the fornix.

Other significant changes were observed in $\mathrm{AD}$ in the $\mathrm{CC}$, which is a major fiber bundle responsible for interhemispheric connection. Using both standard and FW-DTI, large clusters of lower FA were observed in the genu and body with minimal splenial changes, particularly using FW-DTI. Several other studies have similarly reported decreased FA in the CC; however, these studies have shown inconsistent regional changes with $\mathrm{AD}$, with some studies finding changes throughout the $\mathrm{CC}^{53}$ and others reporting predominant changes in the genu $\mathrm{u}^{9}$ or splenium $^{54}$. Early involvement of the genu would support the anterior-to-posterior progression of the retrogenesis hypothesis, wherein degeneration occurs in reverse of myelination. In contrast, early changes in the splenium would be consistent the posterior-to-anterior progression of Wallerian degeneration following neuronal loss, wherein WM damage is subsequent to grey matter damage. To date, conclusive evidence of a gradient pattern has remained elusive ${ }^{55,56}$. Given the substantial FW component observed in the CC, the inclusion of FW correction algorithms could improve accuracy in the resulting DTI metrics in the presence of neurodegenerative changes, and future studies should consider whether the implementation of FW correction could increase confidence in the spatiotemporal patterns observed across DTI studies.

Higher $\mathrm{FA}_{\mathrm{t}}$ in $\mathrm{AD}$ compared with $\mathrm{HC}$ was observed seemingly paradoxically using FW-DTI in bilateral ATR and CST, as well as internal capsule and corona radiata; with standard DTI, much smaller clusters of higher FA were observed in the right hemisphere (versus bilateral for FW-DTI) in the ATR, CST, PLIC and SCR (see Table 2 and Supplementary Tables 2 and 3). In the presence of a unidirectional fiber tract, higher FA would suggest lower $\mathrm{RD}$ and/or higher $\mathrm{AxD}$, which are in contrast to the expected changes in the presence of neurodegeneration. However, one of the major drawbacks of standard DTI methods is an inability to resolve different fiber orientations within a voxel ${ }^{57}$, which arises from the assumption of Gaussian diffusion implicit in the tensor model ${ }^{58}$. In the presence of crossing fibers, FA is artificially diminished due to multi-directional fiber tracts; thus, ADrelated neurodegeneration in these regions may yield increased FA due to the loss of crossing fibers. In these cases, MA - a metric of diffusion anisotropy - may provide insight in areas of crossing fibers; moreover, higher MA has been related to a loss of crossing fibers in early AD pathology in regions with known complicated tract topologies ${ }^{59}$. Further corroborating the presence of fiber crossing, MA was higher in AD subjects in the right CST, PLIC, and SCR. Conversely, lower MA in AD was found in clusters in the left ATR, forceps major and minor, IFOF, UF, genu of CC, anterior CR, and EC. Similar to these results, other studies have found parallel increases of both FA and MA in AD patients in a large cluster extending along the CST and involving the ATR ${ }^{54,60}$. The higher MA in AD subjects, together with higher FA, suggests a more linear shape of the diffusion tensor, indicative of the loss of crossing fiber populations in these areas. As the CST is relatively preserved in AD pathology, these changes suggest degeneration of fiber tracts in the ATR, CC, and fasciculus.

Using standard DTI, non-specific higher $\mathrm{AxD}$ and $\mathrm{RD}$ were observed across $\mathrm{WM}$, while $\mathrm{FW}-\mathrm{AxD}_{\mathrm{t}}$ and FW$\mathrm{RD}_{\mathrm{t}}$ had better agreement with regional $\mathrm{FA}_{t}$ changes. This may be reflective of a larger impact of isotropic $\mathrm{FW}$ diffusion on AxD and RD metrics compared to FA. For example, lower FA in the CC and fornix is indicative of altered WM integrity (e.g., demyelination) in the $\mathrm{AD}$ group; these changes were driven by both higher AxD and RD with standard DTI, while FW correction showed that these changes in $\mathrm{FA}_{\mathrm{t}}$ may be driven by underlying lower $\mathrm{AxD}_{\mathrm{t}}$. Using $\mathrm{FW}$-DTI, lower $\mathrm{FW}-\mathrm{AxD}_{\mathrm{t}}(\mathrm{AD}<\mathrm{HC})$ were found in $\mathrm{WM}$ locations corresponding to lower $\mathrm{FA}_{t}$, while the fornix showed simultaneous lower FW- $\mathrm{RD}_{\mathrm{t}}(\mathrm{HC}>\mathrm{AD})$ in a small cluster. With standard DTI, AxD was higher across multiple regions, consistent with prior literature ${ }^{14,49,54,61,62}$. However, the mechanism of higher $\mathrm{AxD}$ is unknown and contrary to prevailing theories of known $\mathrm{AD}$ pathology ${ }^{61}$. Using FW-DTI, regions of lower $\mathrm{AxD}_{\mathrm{t}}$ corresponded to lower $\mathrm{FA}_{t}$ values, suggesting that prior reports of higher $\mathrm{AxD}$ may have been sensitive to the effects of FW. A similar trend of AxD decreasing with FW correction was previously reported using TBSS in the context of $\mathrm{AD}$ and cerebrovascular disease ${ }^{21}$. After FW correction, higher $\mathrm{AxD}_{\mathrm{t}}$ in $\mathrm{AD}$, compared with $\mathrm{HCs}$, was observed in regions with known crossing fibers, consistent with degeneration of select fiber tracts in these regions. Additionally, clusters with higher FW-FA, values in AD (e.g., ATR, CST, anterior/posterior LIC, and $\mathrm{SFOF}$ ) were associated with higher $\mathrm{FW}-\mathrm{AxD}_{\mathrm{t}}$ and lower $\mathrm{FW}-\mathrm{RD}_{\mathrm{t}}$. Using standard $\mathrm{DTI}$ analysis, no lower $\mathrm{AxD}$ or RD values were detected in $\mathrm{AD}$ subjects.

The FW volume fraction was quantified using the FW-correction algorithm and, consistent with AD-related neurodegeneration, was higher in AD in many WM areas and tracts. More specifically, large clusters of higher FW were located in the cingulum (hippocampal and cingulate gyrus parts, $85.16 \%$ and $56.34 \%$, respectively), ILF and IFOF (52\% and 40\%, respectively), forceps major and minor (approximately $40 \%$ each), and ATR (31.39\%). Similar clusters were also found in the fornix (95.6\%) and CC (genu, body, and splenium; approximately $56 \%$ of the total CC). Additionally, approximately $42 \%$ of the FW clusters were located in WM outside the regions specified by the atlases; in particular, FW increases were found in several regions bordering various sulcal fundi. Consistent with DTI-based metrics, the FW index further demonstrates an important connection between the fornix, $\mathrm{CC}$, and $\mathrm{AD}$. The high volume of $\mathrm{FW}$ in these regions may be related to loss of WM integrity; moreover, the contaminating effects of FW further validates the significant differences observed between standard and FWDTI metrics. This metric may itself be a valuable biomarker, consistent with our hypothesis, as previous studies 
have shown that higher FW values were associated with poorer executive functioning, visual construction, and visuomotor performance ${ }^{21}$. Overall, FW-DTI provides not only more accurate and consistent DTI-based biomarkers, but also provides quantitative FW volume fractions, which may be a potential biomarker of sub-voxel neurodegenerative processes.

Correlations between DTI metrics and various neuropsychology assessments could lend insight into the role of WM changes on cognitive function in AD pathology. In this study, we did not observe any significant voxel-wise correlations between MMSE and DTI metrics before or after FW correction in the AD cohort. This may be attributable to the similar group characteristics (predominantly very mild to mild AD) and the relatively narrow range of MMSE scores, while future studies could consider including more sensitive measures of cognitive functioning, including various sub-domains such as memory and executive function. Despite the lack of correlation in this study, the overall finding that FW-DTI metrics are more consistent with the pathology may lend insight into the inconsistent correlative findings across the literature. While the relationship between WM integrity as assessed by DTI and cognitive functions remains unsettled, the implementation of FW correction to yield more accurate DTI metrics should be considered as a methodological improvement for future correlation studies, particularly for the diffusivity metrics AxD and RD.

There are several limitations to this study. First, we used a voxel-based approach for WM analysis rather than a skeletonized approach, such as TBSS. As TBSS minimizes tract variance across subjects using a common skeletonized tract, it uses fewer voxels and is inherently less sensitive to partial volume effects. However, the higher number of voxels available with voxel-based approaches may increase sensitivity to subtle differences in microstructure ${ }^{63}$. Even so, all of these approaches necessitate the use of atlases, which may be limited in the WM regions that are represented (in this case, the atlases covered $84 \%$ of the WM mask); this is particularly relevant for the FW index that showed widespread changes across WM, even outside of the atlas regions. In the present study, atrophy likely minimal due to the selected patient population, further enabling the use of a voxel-based approach. Second, no significant voxel-based correlations $(p<0.01$ FWE corrected) were observed between standard DTI and FW-DTI metrics and the MMSE score, which is consistent with several previous studies ${ }^{13-16}$. This suggests that while WM is altered in AD, the relationship between global cognitive scores and WM pathology may not be linear. Future work could consider more sensitive measures of cognitive subdomains, and the inclusion of a longitudinal aspect may further pinpoint the temporal ordering of WM alterations relative to GM pathology and cognitive decline. Finally, as early biomarkers are of increasing interest, future studies should also include cohorts of MCI or subjects at high risk of developing AD.

\section{Conclusion}

In conclusion, this study demonstrates that the implementation of a FW correction algorithm for DTI improves both the sensitivity and specificity of derived DTI metrics by removing PVEs, and better captures underlying $\mathrm{AD}$-related pathologic changes than standard DTI approaches. We found significantly altered DTI metrics in AD compared to HC for both standard and FW-DTI, but importantly FW-DTI identified changes were more consistent with known AD pathology as hypothesized (both in terms of magnitude and direction of DTI changes). In addition, MA may improve specificity related to crossing fibers, while the FW index may improve sensitivity to sub-voxel neurodegeneration. Overall, FW-DTI improves the reliability and inter-parameter consistency of DTI metrics in the presence of atrophy, and the resulting metrics provide more sensitive and specific insight into $\mathrm{AD}$-related pathological changes in white matter.

Received: 2 April 2020; Accepted: 9 March 2021

Published online: 26 March 2021

\section{References}

1. Ferri, C. P. et al. Global prevalence of dementia: A Delphi consensus study. Lancet 366(9503), 2112-2117 (2005).

2. Oishi, K. \& Lyketsos, C. G. Alzheimer's disease and the fornix. Front. Aging Neurosci. 6, 241 (2014).

3. Sexton, C. E., Kalu, U. G., Filippini, N., Mackay, C. E. \& Ebmeier, K. P. A meta-analysis of diffusion tensor imaging in mild cognitive impairment and Alzheimer's disease. Neurobiol. Aging 32(12), 2322.e5-18 (2011).

4. Jones, D. K. \& Cercignani, M. Twenty-five pitfalls in the analysis of diffusion MRI data. NMR Biomed. 23(7), 803-820 (2010).

5. Vos, S. B., Jones, D. K., Jeurissen, B., Viergever, M. A. \& Leemans, A. The influence of complex white matter architecture on the mean diffusivity in diffusion tensor MRI of the human brain. Neuroimage 59(3), 2208-2216 (2012).

6. Concha, L. A macroscopic view of microstructure: Using diffusion-weighted images to infer damage, repair, and plasticity of white matter. Neuroscience 276, 14-28 (2014).

7. Liu, Y. et al. Diffusion tensor imaging and tract-based spatial statistics in Alzheimer's disease and mild cognitive impairment. Neurobiol. Aging 32(9), 1558-1571 (2011).

8. Stricker, N. H. et al. Decreased white matter integrity in late-myelinating fiber pathways in Alzheimer's disease supports retrogenesis. Neuroimage 45(1), 10-16 (2009).

9. Ukmar, M. et al. Evaluation of white matter damage in patients with Alzheimer's disease and in patients with mild cognitive impairment by using diffusion tensor imaging. Radiol. Med. 113(6), 915-922 (2008).

10. Bozzali, M. et al. White matter damage in Alzheimer's disease assessed in vivo using diffusion tensor magnetic resonance imaging. J. Neurol. Neurosurg. Psychiatry 72(6), 742-746 (2002).

11. Shim, G. et al. Predicting neurocognitive function with hippocampal volumes and DTI metrics in patients with Alzheimer's dementia and mild cognitive impairment. Brain Behav. 7(9), e00766 (2017).

12. Xue, Y. et al. Characterization of Alzheimer's disease using ultra-high b-values apparent diffusion coefficient and diffusion kurtosis imaging. Aging Dis. 10(5), 1026-1036 (2019).

13. Ibrahim, I. et al. Combination of voxel based morphometry and diffusion tensor imaging in patients with Alzheimer's disease. Neuro Endocrinol. Lett. 30(1), 39-45 (2009).

14. Mayo, C. D. et al. Relationship between DTI metrics and cognitive function in Alzheimer's disease. Front. Aging Neurosci. 10, 436 (2018). 
15. Patil, R. B. \& Ramakrishnan, S. Analysis of sub-anatomic diffusion tensor imaging indices in white matter regions of Alzheimer with MMSE score. Comput Methods Programs Biomed 117(1), 13-19 (2014).

16. Stahl, R. et al. White matter damage in Alzheimer disease and mild cognitive impairment: Assessment with diffusion-tensor MR imaging and parallel imaging techniques. Radiology 243(2), 483-492 (2007).

17. Pierpaoli, C., Jezzard, P., Basser, P. J., Barnett, A. \& Di Chiro, G. Diffusion tensor MR imaging of the human brain. Radiology 201(3), 637-648 (1996).

18. Pasternak, O., Sochen, N., Gur, Y., Intrator, N. \& Assaf, Y. Free water elimination and mapping from diffusion MRI. Magn. Reson. Med. 62(3), 717-730 (2009).

19. Metzler-Baddeley, C., O'Sullivan, M. J., Bells, S., Pasternak, O. \& Jones, D. K. How and how not to correct for CSF-contamination in diffusion MRI. Neuroimage 59(2), 1394-1403 (2012).

20. Hoy, A. R. et al. Microstructural white matter alterations in preclinical Alzheimer's disease detected using free water elimination diffusion tensor imaging. PLoS ONE 12(3), e0173982 (2017).

21. Ji, F. et al. Distinct white matter microstructural abnormalities and extracellular water increases relate to cognitive impairment in Alzheimer's disease with and without cerebrovascular disease. Alzheimers Res. Ther. 9(1), 63 (2017).

22. Fletcher, E., Carmichael, O., Pasternak, O., Maier-Hein, K. H. \& DeCarli, C. Early brain loss in circuits affected by alzheimer's disease is predicted by fornix microstructure but may be independent of gray matter. Front. Aging Neurosci. 6, 106 (2014).

23. Maier-Hein, K. H. et al. Widespread white matter degeneration preceding the onset of dementia. Alzheimers Dement. 11(5), 485-493.e2 (2015).

24. Ofori, E. et al. Free-water imaging of the hippocampus is a sensitive marker of Alzheimer's disease. Neuroimage Clin. 24, 101985 (2019).

25. Arribarat G., O. Pasternak, A. De Barros, M. Galitzky, O. Rascol and P. Peran, Substantia nigra locations of iron-content, free-water and mean diffusivity abnormalities in moderate stage Parkinson's disease. Parkinsonism Relat. Disord. 65, 146-152 (2019). https:// doi.org/10.1016/j.parkreldis.2019.05.033.

26. Bergamino, M., Kuplicki, R., Victor, T. A., Cha, Y. H. \& Paulus, M. P. Comparison of two different analysis approaches for DTI free-water corrected and uncorrected maps in the study of white matter microstructural integrity in individuals with depression. Hum. Brain Mapp. 38(9), 4690-4702 (2017).

27. Bergamino, M., Pasternak, O., Farmer, M., Shenton, M. E. \& Hamilton, J. P. Applying a free-water correction to diffusion imaging data uncovers stress-related neural pathology in depression. Neuroimage Clin. 10, 336-342 (2016).

28. Dumont, M. et al. Free water in white matter differentiates MCI and AD from control subjects. Front. Aging Neurosci. 11, 270 (2019).

29. Marcus, D. S., Fotenos, A. F., Csernansky, J. G., Morris, J. C. \& Buckner, R. L. Open access series of imaging studies: Longitudinal MRI data in nondemented and demented older adults. J. Cogn. Neurosci. 22(12), 2677-2684 (2010).

30. Ardekani, B. A., Bachman, A. H., Figarsky, K. \& Sidtis, J. J. Corpus callosum shape changes in early Alzheimer's disease: An MRI study using the OASIS brain database. Brain Struct. Funct. 219(1), 343-352 (2014).

31. Bachman, A. H., Lee, S. H., Sidtis, J. J. \& Ardekani, B. A. Corpus callosum shape and size changes in early Alzheimer's disease: A longitudinal MRI study using the OASIS brain database. J. Alzheimers Dis. 39(1), 71-78 (2014).

32. Folstein, M. F., Folstein, S. E. \& McHugh, P. R. "Mini-mental state". A practical method for grading the cognitive state of patients for the clinician. J. Psychiatr. Res. 12(3), 189-198 (1975).

33. Khan, T.K. Clinical Diagnosis of Alzheimer's Disease in Biomarkers in Alzheimer's Disease, Elsevier, Editor. (Academic Press, 2016). ISBN 978-0-12-804832-0. https://doi.org/10.1016/C2015-0-04217-4.

34. Smith, S. M. et al. Advances in functional and structural MR image analysis and implementation as FSL. Neuroimage 23(Suppl 1), S208-S219 (2004).

35. Andersson, J. L. R. \& Sotiropoulos, S. N. An integrated approach to correction for off-resonance effects and subject movement in diffusion MR imaging. Neuroimage 125, 1063-1078 (2016).

36. Smith, S. M. Fast robust automated brain extraction. Hum. Brain Mapp. 17(3), 143-155 (2002).

37. Ennis, D. B. \& Kindlmann, G. Orthogonal tensor invariants and the analysis of diffusion tensor magnetic resonance images. Magn. Reson. Med. 55(1), 136-146 (2006).

38. Keihaninejad, S. et al. The importance of group-wise registration in tract based spatial statistics study of neurodegeneration: A simulation study in Alzheimer's disease. PLoS ONE 7(11), e45996 (2012).

39. Avants, B. B., Epstein, C. L., Grossman, M. \& Gee, J. C. Symmetric diffeomorphic image registration with cross-correlation: Evaluating automated labeling of elderly and neurodegenerative brain. Med. Image Anal. 12(1), 26-41 (2008).

40. Varentsova, A., Zhang, S. \& Arfanakis, K. Development of a high angular resolution diffusion imaging human brain template. Neuroimage 91, 177-186 (2014).

41. Winkler, A. M., Ridgway, G. R., Webster, M. A., Smith, S. M. \& Nichols, T. E. Permutation inference for the general linear model. Neuroimage 92, 381-397 (2014).

42. Smith, S. M. \& Nichols, T. E. Threshold-free cluster enhancement: Addressing problems of smoothing, threshold dependence and localisation in cluster inference. Neuroimage 44(1), 83-98 (2009).

43. Hua, K. et al. Tract probability maps in stereotaxic spaces: Analyses of white matter anatomy and tract-specific quantification. Neuroimage 39(1), 336-347 (2008)

44. Wakana, S. et al. Reproducibility of quantitative tractography methods applied to cerebral white matter. Neuroimage 36(3), 630-644 (2007).

45. Hopper, M. W. \& Vogel, F. S. The limbic system in Alzheimer's disease. A neuropathologic investigation. Am. J. Pathol. 85(1), 1-20 (1976).

46. Oishi, K., Mielke, M. M., Albert, M., Lyketsos, C. G. \& Mori, S. The fornix sign: A potential sign for Alzheimer's disease based on diffusion tensor imaging. J. Neuroimaging 22(4), 365-374 (2012).

47. Ringman, J. M. et al. Diffusion tensor imaging in preclinical and presymptomatic carriers of familial Alzheimer's disease mutations. Brain 130(Pt 7), 1767-1776 (2007)

48. Mielke, M. M. et al. Regionally-specific diffusion tensor imaging in mild cognitive impairment and Alzheimer's disease. Neuroimage 46(1), 47-55 (2009).

49. Bosch, B. et al. Multiple DTI index analysis in normal aging, amnestic MCI and AD. Relationship with neuropsychological performance. Neurobiol. Aging 33(1), 61-74 (2012).

50. Damoiseaux, J. S. et al. White matter tract integrity in aging and Alzheimer's disease. Hum. Brain Mapp. 30(4), 1051-1059 (2009).

51. Douaud, G. et al. Brain microstructure reveals early abnormalities more than two years prior to clinical progression from mild cognitive impairment to Alzheimer's disease. J. Neurosci. 33(5), 2147-2155 (2013).

52. Honea, R. A., Vidoni, E., Harsha, A. \& Burns, J. M. Impact of APOE on the healthy aging brain: A voxel-based MRI and DTI study. J. Alzheimers Dis. 18(3), 553-564 (2009).

53. Sun, X. et al. Destruction of white matter integrity in patients with mild cognitive impairment and Alzheimer disease. J. Investig. Med. 62(7), 927-933 (2014).

54. Palesi, F. et al. Specific patterns of white matter alterations help distinguishing Alzheimer's and vascular dementia. Front. Neurosci. $12,274(2018)$ 
55. Alves, G. S. et al. Integrating retrogenesis theory to Alzheimer's disease pathology: Insight from DTI-TBSS investigation of the white matter microstructural integrity. Biomed. Res. Int. 2015, 291658 (2015).

56. Di Paola, M. et al. When, where, and how the corpus callosum changes in MCI and AD: A multimodal MRI study. Neurology 74(14), 1136-1142(2010).

57. Tuch, D. S. et al. High angular resolution diffusion imaging reveals intravoxel white matter fiber heterogeneity. Magn. Reson. Med. 48(4), 577-582 (2002).

58. Alexander, A. L., Hasan, K. M., Lazar, M., Tsuruda, J. S. \& Parker, D. L. Analysis of partial volume effects in diffusion-tensor MRI. Magn. Reson. Med. 45(5), 770-780 (2001).

59. Douaud, G. et al. DTI measures in crossing-fibre areas: Increased diffusion anisotropy reveals early white matter alteration in MCI and mild Alzheimer's disease. Neuroimage 55(3), 880-890 (2011).

60. Teipel, S. J. et al. Fractional anisotropy changes in Alzheimer's disease depend on the underlying fiber tract architecture: A multiparametric DTI study using joint independent component analysis. J. Alzheimers Dis. 41(1), 69-83 (2014).

61. Acosta-Cabronero, J., Alley, S., Williams, G. B., Pengas, G. \& Nestor, P. J. Diffusion tensor metrics as biomarkers in Alzheimer's disease. PLoS ONE 7(11), e49072 (2012).

62. Huang, H. et al. Distinctive disruption patterns of white matter tracts in Alzheimer's disease with full diffusion tensor characterization. Neurobiol. Aging 33(9), 2029-2045 (2012).

63. Preti, M. G. et al. Assessing corpus callosum changes in Alzheimer's disease: Comparison between tract-based spatial statistics and atlas-based tractography. PLoS ONE 7(4), e35856 (2012).

\section{Acknowledgements}

The authors thank Elliott Mufson, PhD, for many helpful discussions. This work was supported by the Barrow Neurological Foundation and the Arizona Alzheimer's Consortium. Data was provided by the OASIS project, supported by the following NIH Grants: P50 AG05681, P01 AG03991, R01 AG021910, P50 MH071616, U24 RR021382, R01 MH56584.

\section{Author contributions}

M.B. and A.M.S. conceived of the idea. M.B. performed data analysis and prepared all figures. M.B. and A.M.S. contributed to interpretation of the results. M.B. and A.M.S. wrote the manuscript, and all authors reviewed the manuscript.

\section{Competing interests}

The authors declare no competing interests.

\section{Additional information}

Supplementary Information The online version contains supplementary material available at https://doi.org/ 10.1038/s41598-021-86505-7.

Correspondence and requests for materials should be addressed to A.M.S.

Reprints and permissions information is available at www.nature.com/reprints.

Publisher's note Springer Nature remains neutral with regard to jurisdictional claims in published maps and institutional affiliations.

(c) (i) Open Access This article is licensed under a Creative Commons Attribution 4.0 International License, which permits use, sharing, adaptation, distribution and reproduction in any medium or format, as long as you give appropriate credit to the original author(s) and the source, provide a link to the Creative Commons licence, and indicate if changes were made. The images or other third party material in this article are included in the article's Creative Commons licence, unless indicated otherwise in a credit line to the material. If material is not included in the article's Creative Commons licence and your intended use is not permitted by statutory regulation or exceeds the permitted use, you will need to obtain permission directly from the copyright holder. To view a copy of this licence, visit http://creativecommons.org/licenses/by/4.0/.

(c) The Author(s) 2021 